\title{
Magnitude of Phenotypic and MTBDRplus Line Probe Assay First-Line Anti-Tuberculosis Drug Resistance Among Tuberculosis Patients; Northwest Ethiopia
}

This article was published in the following Dove Press journal:

Infection and Drug Resistance

\author{
Wubet Birhan Yigzaw' \\ Jordi B Torrelles ${ }^{2}$ \\ Shu-Hua Wang ${ }^{3}$ \\ Belay Tessema'
}

'Department of Medical Microbiology, College of Medicine and Health Sciences, University of Gondar, Gondar, Ethiopia; ${ }^{2}$ Population Health Program, Texas Biomedical Research Institute, San Antonio, TX, USA; ${ }^{3}$ Department of Internal Medicine, Division of Infectious Diseases, College of Medicine, The Ohio State University, Columbus, OH, USA
Correspondence: Wubet Birhan Yigzaw Department of Medical Microbiology, College of Medicine and Health Sciences, University of Gondar, P.O. Box: 196, Gondar, Ethiopia

Email yeshmebetessey@gmail.com
Background: Mycobacterium tuberculosis (Mtb) drug resistance is a key challenge in ending TB.

Objective: The study aimed to determine anti-TB drug resistance and compare the discordance between phenotypic and genotypic drug-susceptibility testing (DST).

Methods: Prospective enrollment and sputum collection from patients suspected of active pulmonary TB from May 2018 to December 2019 at the University of Gondar Hospital. Phenotypic DST study for streptomycin, isoniazid, rifampin, and ethambutol was done by MGIT 360 SIRE Kit. Genotypic resistance for isoniazid and rifampin was performed by MTBDRplus v2 line probe assay (LPA) and compared to phenotypic drug resistance.

Results: A total of 376 patients, median age 32 years, and $53.7 \%$ male were enrolled. $M t b$ was isolated from 126 patients. 106/126 (84\%) patients were newly diagnosed with TB and 20 patients with prior TB treatment. Seventy $(66.0 \%)$ were susceptible to all anti-TB drugs tested. Twenty-five (19.8\%) of the isolates were resistant to isoniazid, $12(9.5 \%)$ to rifampicin and six (5\%) were multidrug resistant. Among previously treated TB patients, $4(20.0 \%)$ and $5(25.0 \%)$ were mono-resistant and poly-resistant, respectively. The sensitivity and specificity of LPA resistance for isoniazid were $94.4 \%$ and $100 \%$, and for rifampin was $75.0 \%$ and $100 \%$, respectively.

Conclusion: The frequency of mono- and poly-drug resistance among both newly diagnosed and previously treated TB patients was high to the rest of the nation. MTBDRplus showed excellent concordance for isoniazid and rifampin. We concluded that DST should be performed for all patients to improve management and decrease spread of drug-resistant $M t b$ strains in the community.

Keywords: Mycobacterium tuberculosis, diagnosis, drug resistance, MGIT, MTBDRplus

\section{Introduction}

Tuberculosis (TB) is among the primary health problems, ${ }^{1,2}$ particularly in lowincome countries, and is considered as the leading cause of death from a single infectious disease ranking above HIV/AIDS. ${ }^{3-9}$ Worldwide, an estimated 10.0 million people felt ill with an estimated 1.4 million deaths in $2019 .{ }^{10}$ Mycobacterium tuberculosis (Mtb) is a grievous pathogenic organism, ${ }^{11}$ infecting nearly one-third of the world's population, where drug-resistant $M t b$ complex strains are expected to account for one third of all deaths due to TB. ${ }^{12,13}$ The 
HIV/AIDS pandemic had significantly diminished public health systems and drove the rise of multidrug-resistant (MDR) and extensive-drug resistant (XDR) TB cases. MDR-TB is caused by strains of $M t b$ that are resistant to at least isoniazid (INH) and rifampicin (RIF). XDR-TB is caused by MDR-Mtb strains that are also resistant to at least one injectable agent (ie, amikacin, kanamycin or capreomycin) and any of the fluoroquinolones. Both MDR- and XDR-TB forms have exacerbated the spread of TB in developing countries. ${ }^{7,14}$

$M t b$ drug resistance is among the key challenges in ending $\mathrm{TB},{ }^{15}$ where the emergence of drug-resistant $\mathrm{TB}$ is delaying its control. ${ }^{16}$ Resistance to INH reduces the probability of treatment success and increases the risk of acquiring resistance to other first-line drugs such as RIF, thereby increasing the risk of MDR-TB. ${ }^{6}$ Current data have shown that INH-resistant TB patients treated with only first-line drugs have poor outcomes. ${ }^{6}$

Recent recommendations for TB drug susceptibility test (DST) comprise both phenotypic and genotypic methods of diagnosis. The solid culture-based DST is the current reference standard of diagnosis of drug resistance, providing results in 4-6 weeks. Other diagnosis methods, such as the rapid automated BACTEC MGIT 960 DST SIRE method (MGIT-SIRE) is also used due to a considerable advantage in shorter turnaround time; ${ }^{17,18}$ however, its cost is sometimes prohibited in low-income areas. Rapid identification of drug resistance is critical to guide proper choice of anti-mycobacterial drugs and to stop the misuse of drugs and subsequent development of additional drug resistance. ${ }^{19,20}$ To overcome limitations of phenotypic methods, such as long turnaround time and high biosafety handling requirements, commercial molecular tests, such as the Line Probe Assay (LPA), have been endorsed by the World Health Organization (WHO). ${ }^{16,17}$

Indeed, quick, accurate and economical methods to detect active MDR-TB cases are urgently needed to ensure that affected patients are identified early, treated correctly, and stay non-infectious. Delayed detection of active MDRTB contributes to suboptimal treatment and poor clinical outcomes, disseminating transmission of MDR-TB. ${ }^{18}$ Consistent DST for isolates of Mtb complex is critical for selecting effective treatment regimens, interruption of transmission, and prevention of further expansion of resistant forms of TB. ${ }^{21}$

Ethiopia is one of the 30 high-TB-burden countries with a current estimated incidence of 140 new TB cases per 100,000 population. ${ }^{10}$ As in other developing countries, TB/HIV co-infection and the emergence of MDR-TB are becoming pressing challenges in the efforts to control TB in Ethiopia. ${ }^{7,22}$ To handle this situation, currently, Ethiopia recommends GeneXpert MTB/RIF as the first-line of DST TB diagnosis and RIF resistance detection in facilities where this molecular diagnosis technique is available. Indeed, in Ethiopia, first-line DST is performed through GeneXpert and MTBDRplus LPA. However, phenotypic DST is not implemented as routine test. This may negatively affect the accurate identification of patients with drug resistance and in turn impact on TB transmission. Thus, the aim of this study was to determine the magnitude of first-line anti-TB drug resistance and compare the discordance between phenotypic and genotypic DST results in the Northwestern of Ethiopia. Our results showed a high frequency of mono- and poly-drug resistance among both newly diagnosed and previously treated TB patients, and urge for the need for a larger evaluation of this problem at national level, especially with the projected devastating impact of the current COVID-19 pandemic on drug-resistant TB diagnosis and treatment. Indeed, MDR-TB has the potential to be exacerbated in the next years due to the saturation of the hospitals and deviation of financial support and resources in low-income countries attending the COVID-19 affected population.

\section{Materials and Methods Study Design}

This Prospective cross-sectional study was conducted from May 2018 to December 2019 at the University of Gondar Hospital TB Directly Observed Treatment, Short Course (DOTS) Clinic and Laboratory, in Gondar, Amhara National Regional State, and Northwest of Ethiopia. This hospital offers service for nearly 5 million inhabitants in the Northwest region of Ethiopia with a catchment area of $45,994 \mathrm{~km}^{2}$. Three hundred seventy six new pulmonary TB patients, with clinical signs and symptoms suggestive of TB and without initiated treatment, visiting the hospital during the study period were eligible for enrollment.

\section{Study Participants Enrollment and Ethical Statement}

Demographic, clinical data and TB risk factors such as prior treatment, recent exposure to TB were collected. After obtaining written informed consent, all study participants provided two sputum samples, one for clinical 
diagnosis using GeneXpert and the other for solid culture using Lowenstein Jensen (LJ) slants. Identification of mycobacteria species was performed by SD Bioline TB Ag MPT64 Rapid test. $M t b$ positive isolates were stored at $-80^{\circ} \mathrm{C}$ until DST was performed. This study was ethically approved by University of Gondar Ethical Review Board (R.No.-O/V/P/RCS/05/387/2016). Permission to conduct this study was also obtained from the University of Gondar Hospital chief clinical director and health center administrators. Written informed consent and assent from legal guardians of children under 18 years were obtained from all study participants, and this study was conducted in accordance with the Declaration of Helsinki. All collected data were kept confidentially decoded for each participant, and samples collected were used only for the intended research.

\section{Drug Susceptibility Test Using MGIT SIRE KIT}

Frozen isolates were retrieved from $-80^{\circ} \mathrm{C}$ and thawed at room temperature. A bacterial suspension was mixed well by vortexing and sub-cultured on BACTEC MGIT liquid media following the manufacturer's instructions. Briefly, $0.8 \mathrm{~mL}$ of PANTA (Polymyxin B, Amphotericin B, Nalidixic Acid, Trimethoprim, and Azlocillin) antibiotic mixture and OADC (Oleic Acid, Bovine Albumin, Dextrose, Catalase) enrichment (Becton Dickinson) was added to MGIT 960 tubes before inoculation, and then $0.5 \mathrm{~mL}$ of the well mixed bacterial suspension was inoculated into MGIT 960 tubes. After inoculation, all the inoculated MGIT 960 tubes were placed in the BACTEC MGIT 960 instrument (Becton Dickinson, Franklin Lakes, $\mathrm{NJ}$, and USA) and incubated at $37^{\circ} \mathrm{C}$ until the instrument flagged positive the MGIT 960 tube. MGIT 960 tubes that gave a positive fluorescent signal were checked for acidfast $M t b$ bacilli using Ziehl-Neelsen staining, whereas MGIT 960 tubes that failed to show any growth within 42 days of incubation were removed and categorized as negative. ${ }^{23}$

Phenotypic DST-SIRE for first-line anti-TB drugs (ST, INH, RIF, and EMB) testing was performed from stored frozen $M t b$ isolates using the BACTEC MGIT 960 DST SIRE method. Lyophilized preparations of the MGIT 960 SIRE kit drugs were reconstituted in $4 \mathrm{~mL}$ sterile distilled water. Final concentrations of STR 1.0 $\mu \mathrm{g} / \mathrm{mL}$, INH $0.1 \mu \mathrm{g} / \mathrm{mL}$, RIF $1.0 \mu \mathrm{g} / \mathrm{mL}$, EMB $5.0 \mu \mathrm{g} /$ $\mathrm{mL}$ were used. A growth control (GC) tube without drugs was included for each isolate. The relative growth ratio between the drug-containing tube and $\mathrm{GC}$ tube was determined by the BD BACTEC MGIT 960 system's software algorithm when 400 growth units for the GC tube were reached. DST results were reported qualitatively. The quality control was maintained by testing the batch of MGIT medium, and SIRE Kit and LPA using the laboratory strain $M t b \mathrm{H}_{37} \mathrm{R}_{\mathrm{v}}$, which is pan-susceptible (susceptible to all anti-TB first-line drugs).

\section{Detection of INH and RIF Resistance Using GenoType ${ }^{\circledR}$ MTBDRplus v2.0}

The identification of genotypic drug resistance to INH (based on mutations in kat $G$ and inhA genes) and RIF (based on mutations the rроB gene) was carried out using GenoType ${ }^{\circledR}$ MTBDRplus assay (MTBDRplus) v2.0 (Hain, Lifescience, Nehren, Germany) based on the manufacturer's instruction in the TB Laboratory at the University of Gondar Hospital TB Laboratory. Accordingly, $50 \mu \mathrm{L}$ of PCR mixture consisting of $10 \mu \mathrm{L}$ AM-A GT MTBDRplus v2.0, $35 \mu \mathrm{L}$ AM-B GT MTBDRplus v2.0 and 5 $\mu \mathrm{L}$ DNA template from a $M t b$ isolate was used to perform this assay. The laboratory reference strain $M t b \mathrm{H}_{37} \mathrm{R}_{\mathrm{v}}$ DNA template was used as a positive control. Water (Qiagen NV, Venlo, the Netherlands, and product) served as a negative control A thermal cycler (VWR, Leicestershire, UK) was programmed as follows: $15 \mathrm{~min}$ for enzyme activation at $95^{\circ} \mathrm{C}$ followed by 10 cycles of $30 \mathrm{sec}$ denaturation at $95^{\circ} \mathrm{C}, 2$ min annealing at $58^{\circ} \mathrm{C}, 20$ cycles of $25 \mathrm{sec}$ denaturation at $95^{\circ} \mathrm{C}, 40 \mathrm{sec}$ annealing at $53^{\circ} \mathrm{C}, 40 \mathrm{sec}$ elongation at $70^{\circ} \mathrm{C}$, and finally elongation at $70^{\circ} \mathrm{C}$ for 8 min. Biotin-labeled amplicons were hybridized to DNA probes attached to a DNA strip. Hybridization was done using the TwinCubator (Hain Lifescience, Nehren, Germany).

Results were interpreted based on the presence and absence of wild-type (WT) and mutation (MUT) probes using the LPA card. ${ }^{24}$ Assays for the identification of mutations in the kat $G$ gene for high-level INH resistance, the inh $A$ gene for low-level INH resistance, and the $r p o B$ gene for RIF resistance were performed on the heat-killed $M t b$ cultures according to the manufacturer's instructions. DNA of the standard laboratory reference $M t b \mathrm{H}_{37} \mathrm{R}_{\mathrm{v}}$ strain and molecular-grade water were used as positive and negative controls, respectively. ${ }^{24}$ 


\section{Data Analysis}

Data were entered and analyzed using SPSS v20 (Statistical Package for the Social Sciences, Chicago, IL, USA). Descriptive statistics were employed. Sensitivity, specificity and positive and negative predictive values including 95\% confidence intervals (CIs) were calculated using SPSS v20 and MedCalc v11.5.1.0 (MedCalc software, Mariakerke, Belgium). Kappa coefficient was also generated. Performance of the MTBDRplus assay in the diagnosis of INH and RIF resistance was evaluated taking results from the MGIT 960 test as reference standard method.

\section{Results}

A total of 376 presumptive pulmonary TB patients were enrolled with median age of 32 years (range: 16-88 years old) and 53.7\% male. A total of $176(46.8 \%)$ sputum samples were culture positive and 10 of $176(5.7 \%)$ were contaminated. Five of $176(2.8 \%)$ sputum samples contained nontuberculosis mycobacteria (NTM). From a total of $161 \mathrm{spu}-$ tum samples positive for $M t b$ complex, isolates were isolated and stored. Phenotypic MGIT SIRE DST tests were done only on $130 \mathrm{Mtb}$ complex isolates due to resources constrains. Of these, 126 (96.9\%) were successfully assessed, while four $(3.1 \%)$ isolates failed because the growth control tube of two of them grew before 4 days of incubation (over growth) with a X400 error. The growth control tube of other two isolates did not grow within 13 days of incubation (under growth) showing a X200 error. Of the successful 126 isolates, we further performed genotypic DST (first-line probe Assay) on 85 culture isolates chosen (Table 1).

Of the 126 patients with Mtb complex isolates tested for phenotypic DST, 106 were newly diagnosed TB patients and 20 patients were previously treated for TB. Among the total Mtb isolates, 43 (34.1\%) showed resistance to at least one drug, $25(19.8 \%)$ were resistant to STR, 25 (19.8\%) for INH, 12 (9.5\%) for RIF, 18 (14.3\%) for EMB and $6(5.0 \%)$ isolates were MDR. Out of the new TB patients, $70(66.0 \%)$ were pan susceptible, $17(16.0 \%)$ were mono resistant, and $21(19.8 \%)$ poly resistant. Among the previously treated TB patients, $13(65.0 \%)$ were pan-susceptible, $4(20.0 \%)$ were mono-resistant, and $5(25.0 \%)$ poly resistant (Table 2 ).

Table I Phenotypic and Genotypic Drug Susceptibility Test (DST) Results

\begin{tabular}{|c|c|c|c|c|}
\hline \multirow[t]{2}{*}{ Variables } & \multicolumn{2}{|c|}{ Phenotypic MGIT DST } & \multicolumn{2}{|c|}{ Genotypic MTBDRplus (LPA) } \\
\hline & Susceptible, n (\%) & Resistance, n (\%) & Susceptible, n (\%) & Resistance, n (\%) \\
\hline \multicolumn{5}{|l|}{ Gender } \\
\hline Male & $48(38.1)$ & $25(19.8)$ & $42(49.4)$ & $7(8.2)$ \\
\hline Female & $34(27.9)$ & $19(15.1)$ & $29(34.1)$ & $7(8.2)$ \\
\hline \multicolumn{5}{|l|}{ Age } \\
\hline $16-30$ years & $49(38.9)$ & $26(20.6)$ & $48(56.5)$ & $7(8.2)$ \\
\hline $31-45$ years & $24(19.1)$ & $15(11.9)$ & $17(20.0)$ & $6(7.1)$ \\
\hline$\geq 46$ years & $9(7.1)$ & $3(2.4)$ & $6(7.1)$ & I (I.28) \\
\hline \multicolumn{5}{|l|}{ Location } \\
\hline Urban & $43(34.1)$ & $24(19.1)$ & $37(43.5)$ & $8(9.4)$ \\
\hline Rural & $39(31.0)$ & $20(15.9)$ & $34(40.0)$ & $6(7.1)$ \\
\hline \multicolumn{5}{|l|}{ HIV status } \\
\hline Negative & $65(51.6)$ & $28(22.2)$ & $54(63.5)$ & $6(7.1)$ \\
\hline Positive & $13(10.3)$ & $14(11.1)$ & $14(16.5)$ & $6(7.1)$ \\
\hline Not known & $4(3.2)$ & $2(1.6)$ & $3(3.5)$ & $2(2.4)$ \\
\hline \multicolumn{5}{|l|}{ TB RX history } \\
\hline New & $69(54.8)$ & $37(29.4)$ & $58(68.2)$ & II (12.9) \\
\hline Relapse & $13(10.3)$ & $7(5.6)$ & $13(15.3)$ & $3(3.5)$ \\
\hline \multicolumn{5}{|l|}{ Risk for TB } \\
\hline No & $63(50.0)$ & $28(22.2)$ & $54(63.5)$ & $9(10.6)$ \\
\hline Yes & $19(15.1)$ & $16(12.7)$ & $17(20.0)$ & $5(5.9)$ \\
\hline
\end{tabular}

Abbreviations: LPA, Line probe assay; MGIT, mycobacterial growth indicator tube; DST, Drug susceptibility pattern; MTBDR, Mycobacterium tuberculosis drug resistance; $\mathrm{RX}$, treatment; TB, tuberculosis. 
Table 2 MGIT 960 SIRE KIT Phenotypic First-Line Anti-TB DST Results of MTBC Isolates

\begin{tabular}{|c|c|c|c|c|c|c|}
\hline \multirow[t]{2}{*}{ TB Cases } & \multirow[t]{2}{*}{ Number of Isolates } & \multicolumn{4}{|c|}{ First-Line Anti-TB Drugs } & \multirow[t]{2}{*}{ Drug Resistance Type Number (\%) } \\
\hline & & INH & RIF & EMB & STR & \\
\hline \multirow[t]{12}{*}{ New $(n=106)$} & $70(66.1)$ & S & S & S & S & Pan-susceptible 70 (66.0) \\
\hline & I (0.9) & S & $\mathrm{R}$ & S & S & Mono-resistance I7 (16.0) \\
\hline & $8(7.6)$ & $\mathrm{R}$ & S & S & S & \\
\hline & $3(2.8)$ & $R$ & $\mathrm{R}$ & $R$ & R & Poly-resistance 19 (17.9) \\
\hline & $8(7.6)$ & S & S & S & $\mathrm{R}$ & \\
\hline & I (0.9) & S & $\mathrm{R}$ & $\mathrm{R}$ & $\mathrm{R}$ & \\
\hline & $4(3.8)$ & $\mathrm{R}$ & S & $\mathrm{R}$ & $\mathrm{R}$ & \\
\hline & $2(1.9)$ & S & $\mathrm{R}$ & S & $\mathrm{R}$ & \\
\hline & I (0.9) & S & $\mathrm{R}$ & $R$ & $S$ & \\
\hline & $4(3.8)$ & $S$ & $S$ & $\mathrm{R}$ & $\mathrm{R}$ & \\
\hline & $3(2.8)$ & $\mathrm{R}$ & S & S & $\mathrm{R}$ & \\
\hline & I (0.9) & $\mathrm{R}$ & $\mathrm{R}$ & S & $\mathrm{R}$ & \\
\hline \multirow[t]{6}{*}{ Re-treatment $(n=20)$} & $13(65.0)$ & $S$ & $S$ & S & S & Pan-susceptible I3 (65.00) \\
\hline & I (5.0) & $S$ & $\mathrm{R}$ & $S$ & S & Mono-resistance 4 (20.00) \\
\hline & $3(15.0)$ & $\mathrm{R}$ & S & S & S & \\
\hline & $\mathrm{I}(5.0)$ & $\mathrm{R}$ & $\mathrm{R}$ & $S$ & S & Poly-resistance 3 (25.00) \\
\hline & I (5.0) & $\mathrm{R}$ & $S$ & $\mathrm{R}$ & $\mathrm{R}$ & \\
\hline & I (5.0) & $R$ & $R$ & S & $\mathrm{R}$ & \\
\hline Any resistance $(n=126)$ & $43(34.1)$ & $25(19.8)$ & $12(9.5)$ & $18(14.3)$ & $28(22.2)$ & MDR $6(5.00)$ \\
\hline
\end{tabular}

Abbreviations: DST, drug sensitivity test; MTBC, Mycobacterium tuberculosis complex; TB, tuberculosis; STR, streptomycin; INH, isoniazid; RIF, rifampicin; EMB, ethambutol; DR type, drug-resistance type; S, susceptible; Pan-S, pan-susceptible; R, resistant; MDR, multidrug resistance.

The number of LPA-based DSTs performed limited the "pairing" of LPA to MGIT SIRE phenotypic DST. However, of the 81 pairs performed, genotypic vs phenotypic DST demonstrated that $63(77.8 \%)$ pairs were susceptible to INH by one of the methods. Of these 63, 17 pairs $(21.0 \%)$ were concordant with INH resistant detected by both methods, and 1 pair $(1.2 \%)$ was discordant showing susceptibility by genotypic and resistance by phenotypic characterization. Conversely, for RIF testing, there were 77 (95.1\%) susceptible and 3 (3.1\%) resistant concordant pairs by both methods, and 1 pair (1.2\%) was discordant showing susceptibility by genotypic and resistance by phenotypic methods. For MDR testing there were 79 concordant pairs (95.1\%) susceptible and 2 concordant pairs $(2.47 \%)$ resistant by both methods, and 1 pair was discordant (1.23\%) showing INH an RIF resistance by genotypic and susceptibility by phenotypic methods. Using the resistant susceptible (RS) and susceptible resistant (SR) pairs, the overall discordance detected was low at $1.23 \%$ (Table 3).

Furthermore, INH LPA compared to MGIT showed the sensitivity of $94.44 \%$ (72.71 to 99.86 ), specificity of $100 \%$ (94.31 to 100 ), positive predictive value of $100 \%$, a negative predictive value of $98.44 \%$ (90.36 to 99.76 ), and accuracy of 0.972 (0.909 to 0.996$)$. RIF LPA testing showed sensitivity of $75 \%$ (19.41 to 99.37), specificity of $100 \%$ (95.32 to 100), a positive predictive value of $100 \%$, a negative predictive value of $98.72 \%$ (93.38 to 99.76), and an accuracy of 0.875 (0.783 to 0.938 ). While for MDR detection LPA demonstrated sensitivity of $100 \%$ (15.81 to 100 ), specificity of $98.75 \%$ (93.23 to 99.97), positive predictive value of $66.67 \%$ (22.19 to 93.35), negative predictive value of $66.67 \%$ (22.19 to 93.35) and accuracy of 0.994 (0.994 to 1.00) (Table 3). The predominant gene mutation 12/17 (70.6\%) for INH resistance was at the codon 315 of katG gene which is a gene coded for high-level resistance (Table 4). 


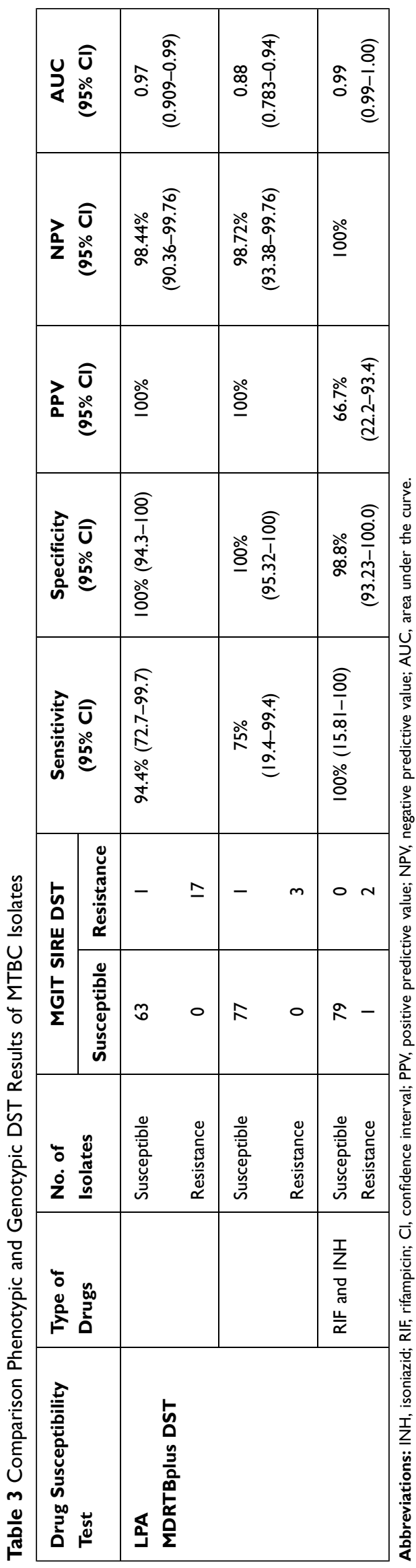

\section{Discussion}

Drug resistance is an important challenge to TB prevention and care especially in low- and middle-income countries. The risk of drug resistance increases due to the inappropriate use of anti-TB drugs. Treatment of MDR-TB is challenging, time-demanding, costly, and often related to a poor treatment outcome. Our result provides updated information on drugresistant TB in both treatment naïve and previously treated individuals. It also highlights the importance of performing drug resistance prior to diagnosis in both groups, particularly for the care and management of patients with drug resistance as well for public health efforts in contact investigation to decrease transmission of drug-susceptible and drug-resistant M. tuberculosis strains.

Ethiopia recommends GeneXpert MTB/RIF as initial diagnostic test and RIF resistance detection in facilities where the equipment is available. First-line LPA is recommended for the defined groups of patients including: Presumptive/confirmed TB patients with prior TB treatment history for one month or more; patients with presumed or confirmed TB with contact history with RR/ MDR-TB; presumptive TB in patients from health care settings or congregated settings or other known high MDR-TB prevalent settings; TB patients who remain smear positive at the end of second months of treatment or later; and TB patients at time of registration to TB treatment if not done as initial diagnosis. ${ }^{25}$

In our study, 43 (34.13\%) were resistant to at least one first-line anti-TB drug, which is lower than other reports performed in Central Ethiopia $(72.9 \%),{ }^{26}$ Mexico $(48.6 \%),{ }^{27}$ and higher than other studies performed in Northwest of Ethiopia (15.8\%), ${ }^{29}$ Vietnam $(26.3 \%),{ }^{28}$ and Myanmar (27.7\%). ${ }^{30}$ The differences in all over prevalence of drug resistance among the different studies could be due to the difference in sample size, study subjects, uneven supply of anti-TB drugs, poor treatment adherence, and poor TB case management.

Although STR is not currently used as part of the firstline anti-TB drug, the highest mono-resistance was found in this anti-TB drug. This may be due to the use of this drug for longer period of time for the treatment of TB and other bacterial infections since its discovery. In this study, INH showed the next highest mono-resistance proportion. This high level of INH mono-resistance may be due to the continuous and/or inappropriate INH preventive therapy in Ethiopia, acquired resistance, and previous INH anti-TB therapy. ${ }^{25}$ Mono-resistance leads to poorer clinical 
Table 4 Frequency of Gene Mutations Associated with Resistance to Isoniazid and Rifampicin by GenoType ${ }^{\circledR}$ MTBDRplus

\begin{tabular}{|l|l|l|l|l|l|}
\hline $\begin{array}{l}\text { Anti-TB } \\
\text { Drugs }\end{array}$ & $\begin{array}{l}\text { Number of Resistant } \\
\text { Isolates }\end{array}$ & $\begin{array}{l}\text { Patterns of Gene Mutations (Wild- } \\
\text { Type/Mutant) }\end{array}$ & $\begin{array}{l}\text { Nucleotide } \\
\text { Change }\end{array}$ & $\begin{array}{l}\text { Amino Acid } \\
\text { Changes }\end{array}$ & $\begin{array}{l}\text { Frequency } \\
(\mathbf{n})\end{array}$ \\
\hline Isoniazid & 17 & $\begin{array}{l}\text { katG WTI/katG MUTI } \\
\text { InhA WTI/InhA MUTI }\end{array}$ & $\begin{array}{l}\text { TCG } \rightarrow \text { ACC } \\
\text { ACC } \rightarrow \text { GCC }\end{array}$ & $\begin{array}{l}\text { Ser3I5ThrI } \\
\text { CI5T }\end{array}$ & $\begin{array}{l}\text { I2 } \\
7\end{array}$ \\
\hline Rifampicin & 3 & $\begin{array}{l}\text { rpoB WT2 and 3 } \\
\text { rpoB WT8 }\end{array}$ & $\begin{array}{l}\text { CTG } \rightarrow \text { ATG } \\
\text { TCG } \rightarrow \text { TTG } \\
\text { CTG } \rightarrow \text { CCG }\end{array}$ & $\begin{array}{l}\text { Leu5II (430)Met } \\
\text { S450L(S43IL) } \\
\text { L452P(L533P) }\end{array}$ & $\begin{array}{l}\text { I } \\
2\end{array}$ \\
\hline
\end{tabular}

Abbreviations: $n$, number of isolates; WT, wild type; MUT, mutant; ND, no mutation detected at mutant probe.

outcomes and prone to the continuation phase therapy with RIF only. Thus, careful attention needs to be given to all TB cases diagnosed being tested for INH resistance.

Interestingly, our finding of MDR TB cases among newly diagnosed TB cases is higher than the Ethiopian national average report of $0.71 \%$, while MDR cases among previously treated TB cases are almost similar $(12 \%)$ with that in the Ethiopian national report. ${ }^{10}$ Twenty three $(21.70 \%)$ isolates were resistant to INH and/or RIF among new cases and six (30\%) of the isolates from previously treated cases were resistant to INH and/or RIF. The greater level of drug resistance among previously treated TB cases when compared to new TB cases might be due to inappropriate drug supply, poor patient adherence to anti-TB treatment regimens, thereby facilitating the selection of spontaneously mutated $M t b$ strains.

The finding of 5\% MDR cases in the current study is only in line with another study performed in the Northwest of Ethiopia, ${ }^{29}$ but lower than other studies performed in Eastern Ethiopia with $6.5 \%,{ }^{31}$ and $10.2 \%,{ }^{32}$ Northwest Ethiopia with $11.7 \%,{ }^{5}$ Myanmar with $18.3 \%,{ }^{30}$ Mexico with $19 \%,{ }^{27}$ Ghana with $36 \%,{ }^{33}$ and higher than in a study done in Vietnam with $1.8 \%{ }^{28}$ In our study, $2.38 \%$ of the isolates were resistant to all first-line antiTB drugs tested (STR, INH, RIF and EMB) and any mono-resistance to STR, INH, RIF and EMB were 28 (22.22\%), 25 (19.84\%), 12 (9.52\%) and 18 (14.29\%), respectively. This is a higher $\%$ than the previous studies in Northwest Ethiopia and Vietnam, ${ }^{28,29}$ but lower than other studies performed in Ethiopia and Mexico. ${ }^{26,27}$ This difference may be due to the difference in study participants, DST methods used, the contribution of health extension workers in Ethiopia, who are working at the community level to increase awareness of patients and supervise drug treatment adherence.
In the GenoType MTBDRplus assay, resistance to INH is identified by probes of the kat $G$ and inhA genes. A higher frequency of resistance to INH occurs due to a mutation of thekat $G$ gene, whereas lower frequency of resistance is caused by mutations in the promoter region of the inh $A$ gene. ${ }^{20}$ Of the 17 INH-resistant isolates, the kat $G$ mutation occurred in $(70.59 \%)$ of the isolates. In all these 12 isolates, specific mutations were found at Codon $\mathrm{S} 315 \mathrm{~T} 1$ of the katG gene, with the mutation pattern of katGWT1/katGMUT1, which were also reported by previous studies conducted in Northwest Ethiopia, ${ }^{34}$ Central Ethiopia, ${ }^{20}$ and South Ethiopia. ${ }^{35}$ Mutations in the inhA gene occurred among 7 (41\%) INH-resistant isolates, which is higher than previous reports from Northwest Ethiopia $^{34}$ and Central Ethiopia. ${ }^{20}$ Specific inhA mutations were found in all seven INH-resistant isolates, which had mutation in Codon C15T. Three isolates were resistant to RIF of which, one isolate presented mutations at rров WT2 and 3 (L511 (430) M), one isolate presented mutations at $r p o B$ WT $8 \mathrm{~S} 450 \mathrm{~L}(\mathrm{~S} 431 \mathrm{~L})$ and another isolate presented mutations at L452P (L533P). ${ }^{36}$

The sensitivity of $94.44 \%$ MTBDRplus assay in detecting INH resistance in our study is in line with previous studies showing $96.3 \%,{ }^{37} 92 \%,{ }^{34}$ but higher than others showing $33.3 \%$, ${ }^{35}$ and $82 \%$ sensitivity. ${ }^{20}$ Whereas in our study, the specificity of $100 \%$ MTBDRplus assay for INH was overall comparable with other studies ranging from $99 \%$ to $100 \% .^{20,34,35,37}$ In our study, MTBDRplus assay showed a sensitivity of $75 \%$ in detecting RIF resistance against MGIT SIRE DST; however, this is lower than previously reported ranging from $80 \%$ to $100 \%$. $^{20,34,37}$ Conversely, our study specificity for RIF $100 \%$ is in agreement with other studies ranging from $99.6 \%$ to $100 \% .^{20,34,35}$ In our study, the sensitivity of MTBDR plus assay for detecting MDR cases was $100 \%$, which is similar to other published studies ranging from $96.4 \%$ to 
$100 \%{ }^{34,35,38}$ However, a lower sensitivity for this test is also reported at $75 \%{ }^{20}$ The specificity of MTBDR plus $98.85 \%$ in our study was in agreement with other studies reporting $100 \% .^{20,34,35,38}$

The concordance of MDRTB plus to all INH, RIF and MDR TB detections in terms of susceptibility testing showed excellent result $(98.77 \%)$, with the low discordance value of $1 / 81(1.23 \%)$ isolates. These results support that LPA can be a good alternative method for detection of INH and RIF resistance, especially where phenotypic DST is not available and a fast treatment decision is required on the MDR status of the patient.

\section{Limitation of the Study}

Small sample size for genotypic DST and we were unable to follow TB treatment outcomes of both drug-resistant and susceptible cases, as well as patients who were classified as clinical cases who received anti-TB drugs without any confirmatory culture results. We did not perform pyrazinamide DST due to the lack of kits.

\section{Conclusions}

A considerable number of new and previously treated TB cases in Northwest Ethiopia harbor both mono-resistant and MDR-TB. Previously treated TB cases showed a considerably higher number of resistances to one or more anti-TB drugs. Phenotypic DST should be started to detect resistance at least to all first-line anti-TB drugs. MTBDRplus LPA showed excellent concordances in terms of sensitivity and specificity detecting monoresistance and MDR TB. The dominance gene mutations related with INH resistance were at the codon 315 of the $k a t G$ gene and codon 15 of $\operatorname{Inh} A$ gene. Our study indicates that the GenoType ${ }^{\circledR}$ MTBDRplus assay has excellent sensitivity and specificity for the diagnosis of resistance to INH, RIF and MDR. Therefore, it could be an alternative method for detection of INH and RIF resistance.

\section{Acknowledgments}

We thank the University of Gondar for financial support to WB and BT, and The Ohio State University, College of Medicine, internal funds to JBT and SHW. We also extend our gratitude to University of Gondar Referral Hospital TB culture laboratory staff, Mr. Yilak Girma, Mrs. Meseret Kassa, Ms. Esseye Derb and Mrs. Ayenesh Abebe for their assistance during the phenotypic and genotypic DST work. The funders had no role in the design and/or analysis of this study.

\section{Disclosure}

The authors report no conflicts of interest for this work.

\section{References}

1. Pitso L, Potgieter S, Van der Spoel van Dijk A. Prevalence of isoniazid resistance-conferring mutations associated with multidrug-resistant tuberculosis in Free State Province, South Africa. S Afr Med J. 2019;109:659-664. doi:10.7196/SAMJ.2019. v109i9.13730

2. Salindri AD, Sales RF, DiMiceli L, et al. Isoniazid monoresistance and rate of culture conversion among patients in the state of Georgia with confirmed tuberculosis, 2009-2014. Ann Am Thorac Soc. 2018;15:331-340. doi:10.1513/AnnalsATS.201702-147OC

3. Sayadi M, Zare H, Jamedar SA, et al. Genotypic and phenotypic characterization of Mycobacterium tuberculosis resistance against fluoroquinolones in the northeast of Iran. BMC Infect Dis. 2020;20:390. doi:10.1186/s12879-020-05112-5

4. Nieto Ramirez LM, Ferro BE, Diaz G, et al. Genetic profiling of Mycobacterium tuberculosis revealed "modern" Beijing strains linked to MDR-TB from Southwestern Colombia. PLoS One. 2020;15:e0224908. doi:10.1371/journal.pone.0224908

5. Lobie TA, Woldeamanuel Y, Asrat D, et al. Genetic diversity and drug resistance pattern of Mycobacterium tuberculosis strains isolated from pulmonary tuberculosis patients in the Benishangul Gumuz region and its surroundings, Northwest Ethiopia. PLoS One. 2020;15:e0231320. doi:10.1371/journal.pone.0231320

6. Jhun BW, Koh WJ. Treatment of isoniazid-resistant pulmonary tuberculosis. Tuberc Respir Dis (Seoul). 2020;83:20-30. doi:10.4046/trd.2019.0065

7. Haile B, Tafess K, Zewude A, et al. Spoligotyping and drug sensitivity of Mycobacterium tuberculosis isolated from pulmonary tuberculosis patients in the Arsi Zone of southeastern Ethiopia. New Microbes New Infect. 2020;33:100620. doi:10.1016/j. nmni.2019.100620

8. Edwards BD, Edwards J, Cooper R, et al. Incidence, treatment, and outcomes of isoniazid mono-resistant Mycobacterium tuberculosis infections in Alberta, Canada from 2007-2017. PLoS One. 2020;15: e0229691. doi:10.1371/journal.pone.0229691

9. Tembo BP, Malangu NG. Prevalence and factors associated with multidrug/rifampicin resistant tuberculosis among suspected drug resistant tuberculosis patients in Botswana. BMC Infect Dis. 2019;19:779. doi:10.1186/s12879-019-4375-7

10. WHO. WHO global tuberculosis report. 2020.

11. Kazemian H, Kardan-Yamchi J, Bahador A, et al. Efficacy of line probe assay in detection of drug-resistant pulmonary tuberculosis in comparison with GeneXpert and phenotypic methods in Iran and genetic analysis of isolates by MIRU-VNTR. Infect Drug Resist. 2019;12:3585-3593. doi:10.2147/IDR.S222905

12. Merker M, Kohl TA, Barilar I, et al. Phylogenetically informative mutations in genes implicated in antibiotic resistance in Mycobacterium tuberculosis complex. Genome Med. 2020;12:27. doi:10.1186/s13073-020-00726-5

13. Swain SS, Sharma D, Hussain T, et al. Molecular mechanisms of underlying genetic factors and associated mutations for drug resistance in Mycobacterium tuberculosis. Emerg Microbes Infect. 2020;9:1651-1663. doi:10.1080/22221751.2020.1785334

14. Gunar G. Multidrug-resistant and extensively drug-resistant tuberculosis: a review of current concepts and future challenges. Clin Med (Northfield Il). 2014;14:279-285. doi:10.7861/clinmedicine.14-3-279

15. Timire C, Metcalfe JZ, Chirenda J, et al. Prevalence of drug-resistant tuberculosis in Zimbabwe: a health facility-based cross-sectional survey. Int $J$ Infect Dis. 2019;87:119-125. doi:10.1016/j. ijid.2019.07.021 
16. Kang JY, Hur J, Kim S, et al. Clinical implications of discrepant results between genotypic MTBDRplus and phenotypic Lowenstein-Jensen method for isoniazid or rifampicin drug susceptibility tests in tuberculosis patients. J Thorac Dis. 2019;11:400-409. doi:10.21037/jtd.2019.01.58

17. Torrea $\mathrm{G}, \mathrm{Ng} \mathrm{KCS}$, Van Deun A, et al. Variable ability of rapid tests to detect Mycobacterium tuberculosis rpoB mutations conferring phenotypically occult rifampicin resistance. Sci Rep. 2019;9:11826. doi:10.1038/s41598-019-48401-z

18. Mahomed S, Mlisana K, Cele L, et al. Discordant line probe genotypic testing vs culture-based drug susceptibility phenotypic testing in TB endemic KwaZulu-Natal: impact on bedside clinical decision making. J Clin Tuberc Other Mycobact Dis. 2020;20:100176. doi:10.1016/j.jctube.2020.100176

19. Hirama T, Sabur N, Derkach P, et al. Risk factors for drug-resistant tuberculosis at a referral centre in Toronto, Ontario, Canada: 2010-2016. Can Commun Dis Rep. 2020;46:84-92. doi:10.14745/ ccdr.v46i04a05

20. Bedewi Omer Z, Mekonnen Y, Worku A, et al. Evaluation of the GenoType MTBDRplus assay for detection of rifampicin- and isoniazid-resistant Mycobacterium tuberculosis isolates in central Ethiopia. Int $J$ Mycobacteriol. 2016;5:475-481. doi:10.1016/j. ijmyco.2016.06.005

21. Yakrus MA, Driscoll J, McAlister A, et al. Molecular and growth-based drug susceptibility testing of Mycobacterium tuberculosis complex for ethambutol resistance in the United States. Tuberc Res Treat. 2016;2016:3404860. doi:10.1155/2016/3404860

22. WHO. WHO global tuberculosis report. 2019.

23. GLI. Mycobacteriology-Laboratory-Manual. First ed. 2014.

24. Hain Lifescience. GenoType MTBDRplus VER 2.0 [Instructions for use]; IFU-304A-06; 2015. Available from: https://www.hain-life science.de/include_datei/kundenmodule/packungsbeilage/download. php?id=936.

25. FMOH. Guidelines for Management of TB, DR-TB and Leprosy in Ethiopia. Sixth ed. 2018.

26. Abate D, Taye B, Abseno M, et al. Epidemiology of anti-tuberculosis drug resistance patterns and trends in tuberculosis referral hospital in Addis Ababa, Ethiopia. BMC Res Notes. 2012;5:462. doi:10.1186/ 1756-0500-5-462

27. Flores-Trevino S, Morfin-Otero R, Rodriguez-Noriega E, et al. Characterization of phenotypic and genotypic drug resistance patterns of Mycobacterium tuberculosis isolates from a city in Mexico. Enferm Infecc Microbiol Clin. 2015;33:181-185. doi:10.1016/j. eimc.2014.04.005
28. Huong N, Lan N, Cobelens F, et al. Antituberculosis drug resistance in the South of Vietnam: prevalence and trends. $J$ Infect Dis. 2006;194:1226-1232. doi:10.1086/507906

29. Tessema B, Beer J, Emmrich F, et al. First- and second-line anti-tuberculosis drug resistance in Northwest Ethiopia. Int J Tuberc Lung Dis. 2012;16:805-811. doi:10.5588/ijtld.11.0522

30. Aung WW, Ei PW, Nyunt WW, et al. Phenotypic and genotypic analysis of anti-tuberculosis drug resistance in Mycobacterium tuberculosis isolates in Myanmar. Ann Lab Med. 2015;35:494-499. doi:10.3343/alm.2015.35.5.494

31. Esmael A, Ali I, Agonafir M, et al. Drug resistance pattern of Mycobacterium tuberculosis in Eastern Amhara Regional State, Ethiopia. J Microb Biochem Technol. 2014;6:75-79. doi:10.4172/ 1948-5948.1000125

32. Brhane M, Kebede A, Petros Y. Molecular detection of multidrug-resistant tuberculosis among smear-positive pulmonary tuberculosis patients in Jigjiga town, Ethiopia. Infect Drug Resist. 2017;10:75-83. doi:10.2147/IDR.S127903

33. Forson A, Kudzawu S, Kwara A, et al. High frequency of first-line anti-tuberculosis drug resistance among persons with chronic pulmonary tuberculosis at a teaching hospital chest clinic. Ghana Med J. 2010;44:42-46. doi:10.4314/gmj.v44i2.68858

34. Belay T, Joerg B, Frank E, et al. Analysis of gene mutations associated with isoniazid, rifampicin and ethambutol resistance among Mycobacterium tuberculosis isolates from Ethiopia. BMC Infect Dis. 2012;12:1-7. doi:10.1186/1471-2334-12-337

35. Wondale B, Medhin G, Abebe G, et al. Phenotypic and genotypic drug sensitivity of Mycobacterium tuberculosis complex isolated from South Omo Zone, Southern Ethiopia. Infect Drug Resist. 2018;11:1581-1589. doi:10.2147/IDR.S165088

36. André E, Goeminne L, Cabibbe A, et al. Consensus numbering system for the rifampicin resistance-associated $\mathrm{rpoB}$ gene mutations in pathogenic mycobacteria. Clin Microbiol Infect. 2017;23:167-172. doi:10.1016/j.cmi.2016.09.006

37. Kebede A, Demisse D, Assefa M, et al. Performance of MTBDRplus assay in detecting multidrug resistant tuberculosis at hospital level. BMC Res Notes. 2017;10:661. doi:10.1186/s13104-017-2989-7

38. Meaza A, Kebede A, Yaregal Z, et al. Evaluation of genotype MTBDRplus VER 2.0 line probe assay for the detection of MDR-TB in smear positive and negative sputum samples. $B M C$ Infect Dis. 2017;17:280. doi:10.1186/s12879-017-2389-6
Infection and Drug Resistance

\section{Publish your work in this journal}

Infection and Drug Resistance is an international, peer-reviewed openaccess journal that focuses on the optimal treatment of infection (bacterial, fungal and viral) and the development and institution of preventive strategies to minimize the development and spread of resistance. The journal is specifically concerned with the epidemiology of antibiotic resistance and the mechanisms of resistance development and diffusion in both hospitals and the community. The manuscript management system is completely online and includes a very quick and fair peerreview system, which is all easy to use. Visit http://www.dovepress.com/ testimonials.php to read real quotes from published authors. 